\title{
Design of a Well-behaved Algorithm for On-board Look-ahead Control
}

\author{
Erik Hellström*, Jan Åslund*, and Lars Nielsen* \\ * Linköping University, Linköping, Sweden, \\ (e-mail: \{hellstrom,jaasl,lars\}@isy.liu.se)
}

\begin{abstract}
:
A look-ahead controller is developed for a heavy diesel truck that utilizes information about the road topography ahead of the vehicle when the route is known. A dedicated prediction model is formulated where special attention is given to properly include gear shifting. The nature of the problem is analyzed for the purpose of optimization, and a well performing dynamic programming algorithm is tailored. A key step for satisfactory solutions with a sufficiently low computational effort is to avoid numerical problems. The focus here is the choice of discretization method, and it turns out that a basic analysis give decisive insight into the interplay between the criterion and the discretization errors. The resulting algorithm is demonstrated to perform well in real on-line tests on a highway.
\end{abstract}

Keywords: Automotive control, dynamic programming, fuel-optimal control.

\section{INTRODUCTION}

A drive mission for a heavy truck is studied, and it is envisioned that there is road data on-board and that the current route is known. In the present work, this information entails the road slope ahead of the vehicle. A drive mission is given by a route, an allowed velocity range and a desired maximum trip time. The objective is to minimize the energy required for a given mission.

Fuel-optimal solutions on basic topographic road profiles are obtained in Schwarzkopf and Leipnik (1977); Monastyrsky and Golownykh (1993); Chang and Morlok (2005); Fröberg et al. (2006). Predictive cruise control is investigated through computer simulations in e.g. Lattemann et al. (2004); Terwen et al. (2004). In Hellström et al. (2006) a predictive cruise controller is also developed where discrete dynamic programming is used to numerically solve the optimal control problem. In Hellström et al. (2007) the approach was evaluated in real experiments.

The purpose of the present paper is to study this problem and to devise a dedicated optimization algorithm. The algorithm should be sufficiently robust and simple in order to enable evaluation on board a vehicle in a real environment. The presentation starts out with a basic analysis that give insights into the nature of the problem. In Section 3, common vehicle models of the longitudinal dynamics including modeling of gear shifts are used to develop a prediction model of the truck. The look-ahead control scheme is explained and the control criterion is defined in the following section. The numerical analysis in Section 5 gives understanding about how the errors due to the discretization method and the criterion interacts. This is a crucial step in the design of the algorithm that in Section 6 are proven to perform well by computer simulation results as well as experimental results from a highway.

\section{A BASIC ANALYSIS}

Consider the motion of a vehicle in one dimension, see Figure 1. The driving force is given by the function $g(u)$

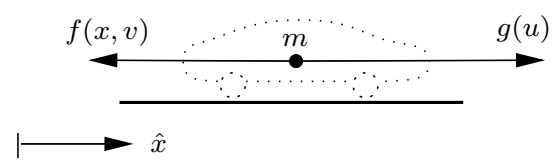

Fig. 1. A vehicle moving in one dimension.

where $u$ is a scalar control variable. The resisting force is dependent on the position $x$ and the velocity $v$ and is denoted by the function $f(x, v)$. It is assumed that this function is monotonically increasing for positive $v$, that is

$$
\frac{\partial f}{\partial v} \geq 0, v>0
$$

which should hold for any physically plausible resistance function. The problem of finding the velocity trajectory that minimizes the work required to move the vehicle from one point $x=0$ to another point $x=s$ is now studied.

Newton's second law of motion in spatial coordinates gives

$$
m v \frac{d v}{d x}=g(u)-f(x, v)
$$

The propulsive work equals

$$
\begin{aligned}
W & =\int_{0}^{s} g(u) d x=\int_{0}^{s}\left(m v \frac{d v}{d x}+f(x, v)\right) d x \\
& =\frac{m}{2}\left(v(s)^{2}-v(0)^{2}\right)+\int_{0}^{s} f(x, v) d x
\end{aligned}
$$


that is, the sum of the difference in kinetic energy and the work due to the resisting force along the path.

The problem objective is now stated as

$$
\min _{v(x)} \int_{0}^{s}\left(m v(x) \frac{d v(x)}{d x}+f(x, v(x))\right) d x
$$

with the time constraint expressed as

$$
\int_{0}^{s} \frac{d x}{v(x)} \leq T
$$

where $T$ denotes the desired maximum time.

If the inequality in (5) is replaced by an equality, the resulting problem is an isoperimetric problem. The core in the calculus of variations is the Euler equation, which for a functional $\int F\left(x, y, y^{\prime}\right) d x$ is

$$
\frac{\partial F}{\partial y}-\frac{d}{d x} \frac{\partial F}{\partial y^{\prime}}=0 .
$$

If the functional has an extremum for a function $y(x)$ but this function does not yield the desired value of another functional $\int G\left(x, y, y^{\prime}\right) d x$, there exist a constant $\lambda$ such that the Euler equation is satisfied for the functional $\int F+\lambda G d x$ (Gelfand and Fomin, 1963).

Only smooth solutions will be considered, so it is assumed that the studied functional has continuous first and second order derivatives in the considered interval for arbitrary $v$ and $v^{\prime}$. In the present problem (4) and (5), the functional

$$
\int_{0}^{s}\left(m v \frac{d v}{d x}+f(x, v)+\frac{\lambda}{v}\right) d x
$$

is formed where $\lambda$ is a constant. Then, according to the Euler equation

$$
m \frac{d v}{d x}+\frac{\partial f}{\partial v}-\frac{d}{d x}(m v)+\lambda\left(-\frac{1}{v^{2}}\right)=0
$$

should be satisfied which yields that

$$
v^{2} \frac{\partial}{\partial v} f(x, v)=\lambda
$$

is a necessary condition for the objective to have an extremum for a function $v(x)$. Due to the assumption (1), the multiplier $\lambda$ will be positive. Relaxing the equality constraint to the inequality (5) does not alter the solution. Every $v(x)$ that becomes admissible when the equality constraint is replaced with an inequality will have a higher value of the objective (4) due to (1).

In order to proceed, assume that the resistance function is a sum of two functions with explicit dependency on $x$ and $v$ respectively, that is

$$
f(x, v)=f_{1}(x)+f_{2}(v) .
$$

The condition (9) then becomes

$$
v^{2} \frac{\partial}{\partial v} f_{2}(v)=\lambda
$$

For a given $\lambda$, the solution to (11) is constant velocity. To minimize the work for moving the body from one point to another point, the extremum is thus a constant speed level adjusted to match the desired trip time.

\subsection{Observations}

For the problem depicted in Figure 1, constant speed is shown to be the solution to the problem of minimizing the needed work to move from one point to another with a trip time constraint. The assumptions are that the velocity and acceleration are smooth and that (1), (2) and (10) holds. However, it is not reasonable to expect that a heavy truck can keep the optimal cruising speed on all road profiles. If the speed can not be kept constant, then it is not plausible that it is possible to always have the same gear engaged either. Therefore, gear selection should be included in the problem description.

\section{TRUCK MODEL}

A model for the longitudinal dynamics of a truck is formulated in this section (Kiencke and Nielsen, 2005).

The engine torque $T_{e}$ is given by $T_{e}=f_{e}\left(\omega_{e}, u_{f}\right)$ where $\omega_{e}$ is the engine speed and $u_{f}$ is the fueling control signal. The function $f_{e}$ is here made up of a lookup table originating from measurements. The clutch, propeller shafts and drive

Table 1. Longitudinal forces.

\begin{tabular}{cll} 
Force & Explanation & Expression \\
\hline$F_{a}(v)$ & Air drag & $\frac{1}{2} c_{w} A_{a} \rho_{a} v^{2}$ \\
$F_{r}(\alpha)$ & Rolling resistance & $m g_{0} c_{r} \cos \alpha$ \\
$F_{N}(\alpha)$ & Gravitational force & $m g_{0} \sin \alpha$ \\
\hline
\end{tabular}

shafts are assumed stiff. The resulting conversion ratio of the transmission and final drive $i(g)$ and their efficiency $\eta(g)$ are functions of the engaged gear number, denoted by $g$. The models of the resisting forces are explained in Table 1 . The relation

$$
v=r_{w} \omega_{w}=\frac{r_{w}}{i(g)} \omega_{e}
$$

is assumed to hold where $r_{w}$ is the wheel radius. When a gear is engaged, this gives

$$
\begin{aligned}
& \frac{d v}{d t}(x, u, \alpha)= \\
& \frac{r_{w}}{J_{l}+m r_{w}^{2}+\eta(g) i(g)^{2} J_{e}}\left(i(g) \eta(g) T_{e}\left(v, u_{f}\right)\right. \\
& \left.-T_{b}\left(u_{b}\right)-r_{w}\left(F_{a}(v)+F_{r}(\alpha)+F_{l}(\alpha)\right)\right)
\end{aligned}
$$

where $x=[v, g]^{T}, u=\left[u_{f}, u_{b}, u_{g}\right]^{T}$ denote the state and control vector respectively. The states are the velocity $v$ and currently engaged gear $g$ and the controls are fueling $u_{f}$, braking $u_{b}$ and gear $u_{g}$. The road slope is denoted by $\alpha$ and the brake torque is denoted by $T_{b}$. All model parameters are explained in Table 2.

Table 2. Prediction model parameters.

\begin{tabular}{clcl}
\hline$J_{l}$ & Lumped inertia & $c_{w}$ & Air drag coefficient \\
$J_{e}$ & Engine inertia & $A_{a}$ & Cross section area \\
$m$ & Vehicle mass & $\rho_{a}$ & Air density \\
$r_{w}$ & Wheel radius & $c_{r}$ & Rolling resistance coefficient \\
$g_{0}$ & Gravity constant & & \\
\hline
\end{tabular}




\subsection{Fuel Consumption}

The mass flow of fuel $\dot{m}$ is determined by the fueling level $u_{f}$ and the engine speed $\omega_{e}$. Assuming again that (12) holds, the mass flow is

$$
\dot{m}(x, u)=\frac{n_{c y l}}{2 \pi n_{r}} \frac{i(g)}{r_{w}} v u_{f}
$$

where $n_{c y l}$ is the number of cylinders and $n_{r}$ is the number of engine revolutions per cycle.

\subsection{Gear Shift Modeling}

It is assumed that the transmission is of the automated manual type and that gear shifts are carried out by engine control. In order to engage neutral gear without using the clutch, the transmission should first be controlled to a state where no torque is transmitted. The engine torque should then be controlled to a state where the input and output revolution speeds of the transmission are synchronized when the new gear is engaged.

Approach In the case of a truck with a large vehicle mass, the time without engine propulsion becomes significant. Assume that the control signal $u_{g}$ changes value from $g_{1}$ to $g_{2}$ at $t=0$ and thereby commands a shift. The currently engaged gear $g(t)$ is then described by

$$
g(t)=\left\{\begin{array}{l}
g_{1}, t<0 \\
0, \quad 0 \leq t \leq \tau \\
g_{2}, t>\tau
\end{array}\right.
$$

where gear zero corresponds to neutral gear. The time in neutral gear, denoted by $\tau$, is assumed constant. Fueling is required to synchronize the engine speed with the corresponding speed of the next gear in case of a downshift. When neutral gear is engaged,

$$
J_{e} \dot{\omega}_{e}=T_{e}=f_{e}\left(\omega_{e}, u_{f}\right)
$$

holds where $J_{e}$ is the engine inertia. It is here assumed that the control $u_{f}$ used is known. The time required for synchronization is then found by simulating (16).

Neutral Gear Modeling When neutral gear is engaged $g=0$, the engine transmits zero torque to the driveline. The ratio $i$ and efficiency $\eta$ are undefined since the engine is decoupled from the rest of the powertrain. The approach taken here is to define the ratio and efficiency of neutral gear to be zero. Then, Equation (13) with $i(0)=\eta(0)=0$ describes the vehicle motion. When neutral gear is engaged, it is assumed that the control $u_{f}$ is at a constant level that gives the desired idle speed of the engine.

\section{LOOK-AHEAD CONTROL}

Look-ahead control is a predictive control scheme with additional knowledge about some of the future disturbances to the controlled system. In the current application, this additional knowledge includes the road topography ahead of the vehicle. An optimization is then performed with respect to a criterion that involves predicted future behavior of the system. This is accomplished through dynamic programming (DP) (Bellman and Dreyfus, 1962).
The prediction model (13)- (16) is discretized in order to obtain a discrete process model

$$
x_{k+1}=f\left(x_{k}, u_{k}, \alpha_{k}\right)
$$

where $x_{k}, u_{k}$ denotes the state and control vectors. The known disturbance, the road slope, is denoted by $\alpha_{k}$. Divide the distance of the entire drive mission into $M$ steps. The problem faced is to find

$$
J_{0}^{*}\left(x_{0}\right)=\min _{u_{0}, \ldots, u_{M-1}} \zeta_{M}\left(x_{M}\right)+\sum_{k=0}^{M-1} \zeta\left(x_{k}, u_{k}, \alpha_{k}\right)
$$

where $\zeta$ and $\zeta_{M}$ defines the running and the terminal cost respectively. A look-ahead horizon is constructed by truncating the entire drive mission horizon of $M$ steps to $N<M$ steps and approximating the cost-to-go at stage $N$. The shorter horizon is used in the online optimization. Rewrite the problem (17) as

$$
\begin{aligned}
J_{0}^{*}\left(x_{0}\right)= & \min _{u_{0}, \ldots, u_{M-1}}\left\{\zeta_{M}\left(x_{M}\right)+\sum_{k=0}^{M-1} \zeta\left(x_{k}, u_{k}, \alpha_{k}\right)\right\} \\
= & \min _{u_{0}, \ldots, u_{N-1}}\left\{\sum_{k=0}^{N-1} \zeta\left(x_{k}, u_{k}, \alpha_{k}\right)\right. \\
& \left.+\min _{u_{N}, \ldots, u_{M-1}}\left\{\zeta_{M}\left(x_{M}\right)+\sum_{k=N}^{M-1} \zeta\left(x_{k}, u_{k}, \alpha_{k}\right)\right\}\right\}
\end{aligned}
$$

and define the residual cost

$$
J_{N}^{*}\left(x_{N}\right)=\min _{u_{N}, \ldots, u_{M-1}} \zeta_{M}\left(x_{M}\right)+\sum_{k=N}^{M-1} \zeta\left(x_{k}, u_{k}, \alpha_{k}\right)
$$

as the cost-to-go function at stage $N$. Replace this function with an approximation $\tilde{J}_{N}^{*}\left(x_{N}\right)$ that should be available at a low computational effort. The problem is now only defined over the look-ahead horizon and

$$
\min _{u_{0}, \cdots, u_{N-1}} \tilde{J}_{N}^{*}\left(x_{N}\right)+\sum_{k=0}^{N-1} \zeta\left(x_{k}, u_{k}\right)
$$

is to be solved. The control $u_{0}$ is applied to the system and the procedure restarts with a horizon that has moved forward in order to calculate the next control according to

$$
\min _{u_{1}, \cdots, u_{N}} \tilde{J}_{N+1}^{*}\left(x_{N+1}\right)+\sum_{k=1}^{N} \zeta\left(x_{k}, u_{k}\right)
$$

where the control $u_{1}$ is applied. This procedure is then repeated.

\subsection{Criterion}

The current objective is to minimize the energy for a given drive mission. Denote by $M$ the fuel use and by $T$ the time required for a trip from $s=s_{0}$ to $s=s_{f}$,

$$
M=\int_{s_{0}}^{s_{f}} \dot{m}(x, u) \frac{d s}{v}, \quad T=\int_{s_{0}}^{s_{f}} \frac{d s}{v} .
$$

To weigh fuel and time use, the criterion chosen is

$$
I=M+\beta T
$$


where $\beta$ is a scalar parameter which can be tuned to receive the desired trade off. The parameter is here chosen in order to receive a stationary solution at a desired cruising speed on flat road for the model (13).

Running Cost The running cost is chosen from the continuous criterion (22) as

$$
\zeta\left(x_{k}, u_{k}, \alpha_{k}\right)=m_{k}+\beta t_{k}
$$

where

$$
m_{k}=\int_{k h}^{(k+1) h} \dot{m}(x, u) \frac{d s}{v}, \quad t_{k}=\int_{k h}^{(k+1) h} \frac{d s}{v} .
$$

Residual cost Known methods for approximating the residual cost (18) involve offline and online calculations (Bertsekas, 2005). One offline approach is to simplify the present model and use it for deriving the approximation. Online calculations can e.g. be based on a heuristic control law where the cost is computed analytically or through simulations.

The residual cost function will here be constructed offline under simplifying assumptions. The disturbance $\alpha_{k}$, the road slope, is assumed to be zero for the next $N$ steps and the cost-to-go with these conditions is calculated. The residual cost $J_{N}^{*}\left(x_{N}\right)$, defined in (18) is thus approximated by $\tilde{J}_{N}^{*}\left(x_{N}\right)$, where

$$
\tilde{J}_{N}^{*}\left(x_{N}\right)=\min _{u_{N}, \ldots, u_{2 N-1}} \sum_{k=N}^{2 N-1} \zeta\left(x_{k}, u_{k}, 0\right) .
$$

The running cost $\zeta$ is given in (23) and the terminal cost $\zeta_{M}$ is here chosen to zero. This approximation is obviously not dependent on the current position and therefore, the solution to (25) can be obtained offline and stored as a tabulated function.

\subsection{Dynamic Programming Algorithm}

Denote by $U_{k}$ the set of allowed controls and by $S_{k}$ the set of allowed states at stage $k$. The DP solution to the look-ahead problem (19) is as follows.

(1) For $x \in S_{N}$, let $J_{N}(x)=\tilde{J}_{N}^{*}(x)$.

(2) Let $k=N-1$.

(3) For $x \in S_{k}$, let

$$
J_{k}(x)=\min _{u \in U_{k}}\left\{\zeta\left(x, u, \alpha_{k}\right)+J_{k+1}\left(f\left(x, u, \alpha_{k}\right)\right)\right\} .
$$

(4) Repeat (3) for $k=N-2, N-3, \ldots, 0$.

(5) The solution is made up of the control with the associated optimal cost $J_{0}^{*}\left(x_{0}\right)=J_{0}\left(x_{0}\right)$.

\section{DISCRETIZATION ANALYSIS}

Performing numerical optimization of dynamical systems inevitable leads to errors such as rounding and truncation errors. It is of course desirable, but hard to guarantee, that such errors do not lead to that the numerical solution differ from the true solution to the original problem.

The choice of discretization method affects algorithm complexity, and two well known and simple methods for solving ordinary differential equations are the Euler forward and backward method. The Euler methods are used in the optimization formulation due to their simplicity.

In the following first some potential problems that can occur are presented. These must be avoided, and therefore test problems and guiding examples are analyzed to accomplish this.

\subsection{Potential Discretization Problems}

Potential problems caused by discretization errors are presented in the following setting. Flat road is assumed for these examples. The shown solutions are obtained by applying the DP algorithm with a grid of 20 steps of $50 \mathrm{~m}$ and a velocity quantization of $0.2 \mathrm{~km} / \mathrm{h}$.

Oscillating solutions are obtained when the Euler forward method is used to discretize the model equations. Figure 2 shows characteristic appearance. The forward method applied to the prediction model is stable for the step lengths used so a stability analysis can not explain the behavior. The oscillating solution obtained when using Euler forward is shown to the left. The more stable solution to the right is obtained with Euler backward. Using a
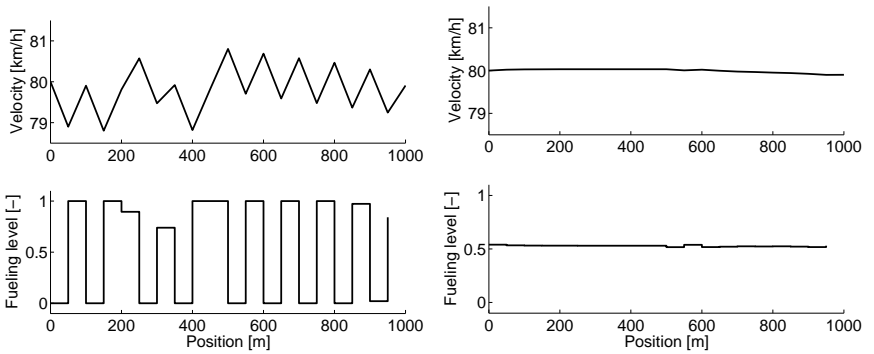

Fig. 2. Using different methods to discretize the model used for optimization. Left: Euler forward. Right: Euler backward.

finer grid does not remove the oscillating behavior, but the amplitude decreases.

\subsection{Basic Analysis Continued}

The vehicle in Figure 1, with one additional assumption, will be used as a test problem. The resisting force is assumed to be independent of $x$, that is $f(x, v)=f(v)$. A model for the system is given by (2). The objective considered is to minimize the work needed to bring the system from $x=0, v(0)=v_{0}$ to $x=s, v(s)=v_{0}$. According to (3), the work needed is

$$
W=\int_{0}^{s} g(u) d x=\int_{0}^{s} f(v) d x
$$

since the kinetic energy at the start and the end of the interval is the same. The time is constrained by $\int_{0}^{s} \frac{d x}{v} \leq T$.

Assume now that the integration interval is subdivided into only three mesh points $0<h<2 h$ where $h$ denotes the step size. The control $u$ is assumed constant on each subinterval,

$$
u(x)=\left\{\begin{array}{l}
u_{0}, 0 \leq x<h \\
u_{1}, h \leq x<2 h
\end{array}\right.
$$


The objective can then be stated as

$$
J=\min _{u_{0}, u_{1}} h\left(g\left(u_{0}\right)+g\left(u_{1}\right)\right)
$$

using Equation (26) and where $v(0)=v_{0}, v(h)=v_{1}$. The maximum time $T$ is chosen as $T=\frac{2 h}{v_{0}}$. The expected solution to the discretized problem is constant speed, that is $v_{1}=v_{0}$ according to Section 2 .

\subsection{Interplay between Criterion and Errors}

The forward Euler method applied on the model (2) gives

$$
\frac{v_{i+1}-v_{i}}{h}=\frac{d v}{d x}\left(x_{i}\right)=\frac{1}{m v_{i}}\left(g\left(u_{i}\right)-f\left(v_{i}\right)\right)
$$

and Euler backward

$$
\frac{v_{i+1}-v_{i}}{h}=\frac{d v}{d x}\left(x_{i+1}\right)=\frac{1}{m v_{i+1}}\left(g\left(u_{i}\right)-f\left(v_{i+1}\right)\right) \text {. }
$$

Now, study the value of the objective (27) when using these different methods. Letting $i=\{0,1\}$, the equations (28) and (29) can be solved for $g\left(u_{0}\right)$ and $g\left(u_{1}\right)$ in each case. Due to the terminal constraints, $v_{2}=v_{0}$. Insertion into the objective (27) gives when using the forward Euler method,

$$
W_{E F}\left(v_{0}, v_{1}\right)=h\left(f\left(v_{0}\right)+f\left(v_{1}\right)\right)-m\left(v_{1}-v_{0}\right)^{2}
$$

and when using the backward Euler method,

$$
W_{E B}\left(v_{0}, v_{1}\right)=h\left(f\left(v_{0}\right)+f\left(v_{1}\right)\right)+m\left(v_{1}-v_{0}\right)^{2} .
$$

Note that $f\left(v_{1}\right)>f\left(v_{0}\right)$ for $v_{1}>v_{0}$ holds by the assumption (1).

The minimum of the objective is expected to occur for $v_{1}=v_{0}$. Therefore,

$$
W_{E F}\left(v_{0}, v_{0}\right)<W_{E F}\left(v_{0}, v_{1}\right) \quad \forall v_{1}>v_{0}
$$

should hold. By inserting (30) into (32),

$$
\frac{f\left(v_{1}\right)-f\left(v_{0}\right)}{v_{1}-v_{0}}>m \frac{v_{1}-v_{0}}{h}
$$

is obtained. Approximating the differences with the corresponding derivatives yields

$$
\frac{d f}{d v}>m \frac{d v}{d s} .
$$

Let the resisting function $f$ correspond to air drag, i.e. $f(v)=\frac{1}{2} c_{w} A_{a} \rho_{a} v^{2}$ where $c_{w}$ is the air drag coefficient, $A_{a}$ is a cross section area and $\rho_{a}$ is the air density. For typical values of a heavy truck with $m=40 \cdot 10^{3} \mathrm{~kg}$ at $v=20 \mathrm{~m} / \mathrm{s}$, $c_{w} A_{a} \rho_{a} \approx 0.6 \cdot 10 \cdot 1.2=7.2<8$. Then according to $(34)$,

$$
\frac{d v}{d t}=v \frac{d v}{d s}<\frac{c_{w} A_{a} \rho_{a} v^{2}}{m}<\frac{8 \cdot 20^{2}}{40 \cdot 10^{3}}=0.08 \mathrm{~m} / \mathrm{s}^{2}
$$

should hold but a greater maximum acceleration is not unreasonable for such a truck at the considered velocity.

When using the Euler backward method (31), it is seen that there is no $v_{1}>v_{0}$ such that the objective becomes lower than when $v_{1}=v_{0}$. Thus, only the Euler backward method guarantee that the solution to the test problem is preserved.
Also note that if the same dynamical model is used with an objective of maximizing instead of minimizing the criterion (26), the Euler forward method would not cause trouble but the Euler backward might.

Alternative Problem Description For the test problem, an alternative problem description can be achieved by using that

$$
\frac{d v}{d t}=v \frac{d v}{d x}=\frac{1}{2} \frac{d}{d x} v^{2}
$$

By introducing the state $y=\frac{1}{2} m v^{2}$ of kinetic energy instead of the velocity $v$, the model

$$
\frac{d y}{d x}=g(u)-f\left(\sqrt{\frac{2}{m} y}\right)
$$

is obtained. The objective value (27) then becomes

$$
W_{E F}=W_{E B}=h\left(f\left(v_{0}\right)+f\left(v_{1}\right)\right)
$$

regardless of which method that is used. Reformulating the problem may thus be beneficial. Applying a higher order method, such as a two-step Runge-Kutta, on the original problem also avoid these numerical problems according to our experience.

\section{REALIZATION}

By avoiding numerical problems, it is possible to devise a well performing DP algorithm that is used in a predictive control strategy. This look-ahead control strategy is evaluated through computer simulations as well as in a demonstrator vehicle. The software needed for the controller is run on a standard portable computer. With the used algorithm parameters, a solution is calculated in tenths of a second.

A demonstrator vehicle was developed in collaboration with SCANIA. More details of these experiments are given in Hellström et al. (2007). The trial route is a $120 \mathrm{~km}$ segment of a Swedish highway. In average, the fuel consumption is decreased about 3.5\% without increasing the trip time and the number of gear shifts is decreased with $42 \%$ traveling back and forth, compared to the standard cruise controller. The tractor and trailer have a gross weight of about 40 tonnes. In Figure 3, measurements from a $2.5 \mathrm{~km}$ segment of the trial route are shown. The look-ahead controller performs well in the sense that the optimal velocity trajectory is rather smooth and intuitive and, compared to the cruise controller, a gear shift is avoided.

To further demonstrate the gain of properly including gear shifts in the prediction model, simulation results presented in the following pinpoint the advantages.

\subsection{Shifting Behavior}

The DP algorithm is let to control a simulation model of a heavy truck. The parameters are set to resemble a typical heavy truck with a gross weight of 40 tonnes. With two choices of gear shift times $\tau=\{0.0,1.0\}$ s in Equation (15), simulation results on road data from a $4.5 \mathrm{~km}$ segment of a Swedish highway are shown in Figure 4. The velocity 

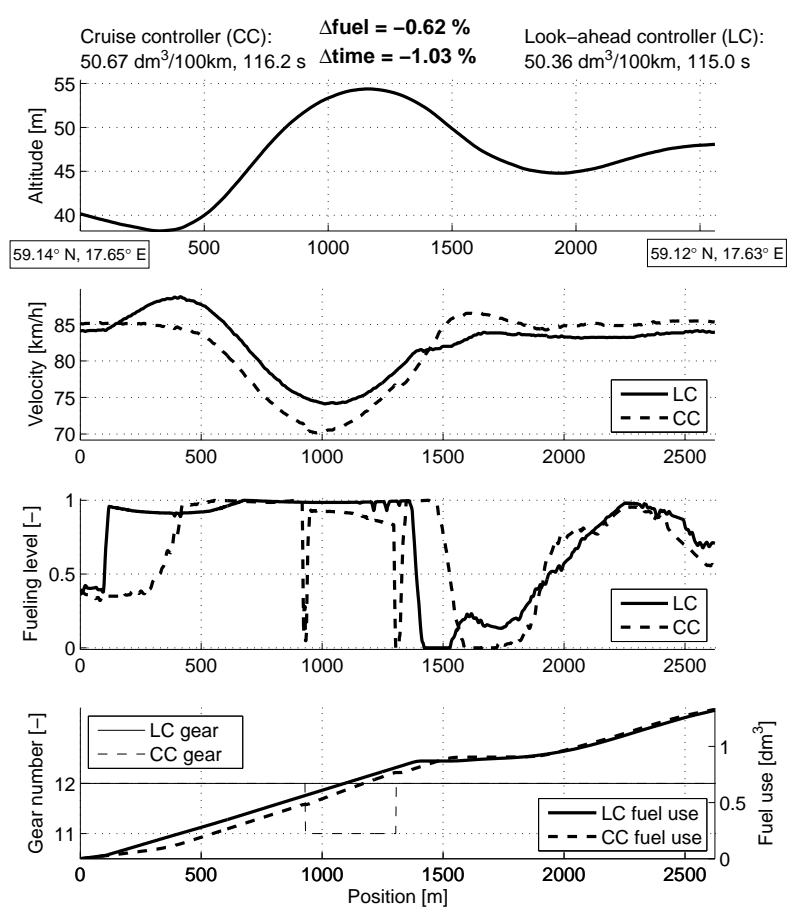

Fig. 3. Measured data from one road segment. The LC gains speed at $200 \mathrm{~m}$ prior to the uphill and avoids a gear shift. At $1400 \mathrm{~m}$ the LC slows down and the truck is let to accelerate in the downslope.

trajectories are similar, but with $\tau=1.0 \mathrm{~s}$, the number of gear shifts is reduced from six to three by shifting several steps. The values of the simulated fuel and time use are close, but the gain is the shifting behavior.
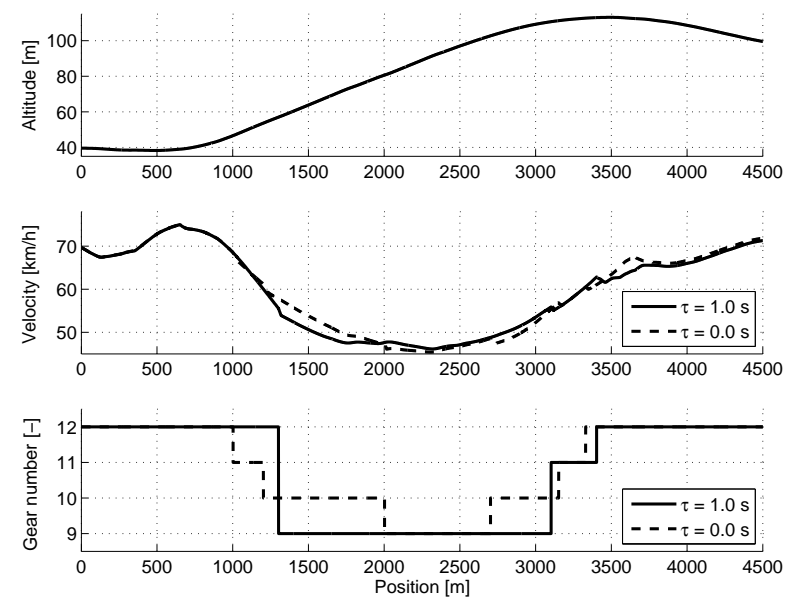

Fig. 4. Simulations of different control trajectories.

The gear shift model is simple but clearly gives different, and more intuitive, controller characteristics. The loss of speed and the increase of the criterion due to a shift is captured in a way that can not be done by e.g. including a penalty term on shifts in the criterion.

\section{CONCLUSIONS}

By a basic analysis of a general longitudinal vehicle model, it was shown that with few assumptions, constant speed is optimal. However, due to the large mass of a heavy truck, it is not possible to keep a desired cruising speed and the loss of propulsion force when shifting gear has a noteworthy influence on vehicle motion. The mass is therefore the most important parameter in the current context and causes a challenging optimization problem.

An analysis has been carried out to avoid discretization problems in the DP algorithm. Oscillating solutions may appear despite established stability and convergence properties of the discretization method. The interplay between the objective and the errors was shown to be crucial. A key step in the design of a well performing algorithm was to avoid problems due to discretization errors, and this was achieved by insights from the analysis.

The algorithm gives satisfactory solutions with a sufficiently low computational complexity and this allowed experimental evaluation via realization in a demonstrator vehicle. The experimental results confirm that, by lookahead control, it is possible to reduce the fuel consumption without increasing the travel time.

\section{REFERENCES}

R.E. Bellman and S.E. Dreyfus. Applied Dynamic Programming. Princeton University Press, Princeton, New Jersey, 1962.

D. P. Bertsekas. Dynamic programming and suboptimal control: A survey from ADP to MPC. European Journal of Control, 11, 2005.

D. J. Chang and E. K. Morlok. Vehicle speed profiles to minimize work and fuel consumption. Journal of transportation engineering, 131(3):173-181, 2005.

A. Fröberg, E. Hellström, and L. Nielsen. Explicit fuel optimal speed profiles for heavy trucks on a set of topographic road profiles. Number 2006-01-1071 in SAE World Congress, Detroit, MI, USA, 2006.

I.M. Gelfand and S.V. Fomin. Calculus of variations. Prentice-Hall, Englewood Cliffs, New Jersey, 1963.

E. Hellström, A. Fröberg, and L. Nielsen. A real-time fuel-optimal cruise controller for heavy trucks using road topography information. Number 2006-01-0008 in SAE World Congress, Detroit, MI, USA, 2006.

E. Hellström, M. Ivarsson, J. Åslund, and L. Nielsen. Look-ahead control for heavy trucks to minimize trip time and fuel consumption. 5th IFAC Symposium on Advances in Automotive Control, Monterey, CA, USA, 2007.

U. Kiencke and L. Nielsen. Automotive Control Systems, For Engine, Driveline, and Vehicle. Springer-Verlag, Berlin, 2nd edition, 2005.

F. Lattemann, K. Neiss, S. Terwen, and T. Connolly. The predictive cruise control - a system to reduce fuel consumption of heavy duty trucks. SAE Technical paper series 2004-01-2616, 2004.

V.V Monastyrsky and I.M. Golownykh. Rapid computations of optimal control for vehicles. Transportation Research, 27B(3):219-227, 1993.

A.B. Schwarzkopf and R.B. Leipnik. Control of highway vehicles for minimum fuel consumption over varying terrain. Transportation Research, 11(4):279-286, 1977.

S. Terwen, M. Back, and V. Krebs. Predictive powertrain control for heavy duty trucks. 4th IFAC Symposium on Advances in Automotive Control, Salerno, Italy, 2004. 